\title{
Associations of thigh muscle fat infiltration with isometric strength measurements based on chemical shift encoding-based water-fat magnetic resonance imaging
}

Stephanie Inhuber ${ }^{1 *}$ D , Nico Sollmann², Sarah Schlaeger ${ }^{2,3}$, Michael Dieckmeyer $^{2,3}$, Egon Burian², Caroline Kohlmeyer ${ }^{1}$, Dimitrios C. Karampinos ${ }^{3}$, Jan S. Kirschke², Thomas Baum² ${ }^{2}$ Florian Kreuzpointner ${ }^{1}$ and Ansgar Schwirtz ${ }^{1}$

\begin{abstract}
Background: Assessment of the thigh muscle fat composition using magnetic resonance imaging (MRI) can provide surrogate markers in subjects suffering from various musculoskeletal disorders including knee osteoarthritis or neuromuscular diseases. However, little is known about the relationship with muscle strength. Therefore, we investigated the associations of thigh muscle fat with isometric strength measurements.

Methods: Twenty healthy subjects (10 females; median age 27 years, range 22-41 years) underwent chemical shift encoding-based water-fat MRI, followed by bilateral extraction of the proton density fat fraction (PDFF) and calculation of relative cross-sectional area (relCSA) of quadriceps and ischiocrural muscles. Relative maximum voluntary isometric contraction (relMVIC) in knee extension and flexion was measured with a rotational dynamometer. Correlations between PDFF, relCSA, and relMVIC were evaluated, and multivariate regression was applied to identify significant predictors of muscle strength.
\end{abstract}

Results: Significant correlations between the PDFF and relMVIC were observed for quadriceps and ischiocrural muscles bilaterally ( $p=0.001$ to 0.049 ). PDFF, but not relCSA, was a statistically significant $(p=0.001$ to 0.049$)$ predictor of relMVIC in multivariate regression models, except for left-sided relMVIC in extension. In this case, PDFF $(p=0.005)$ and relCSA ( $p=0.015$ ) of quadriceps muscles significantly contributed to the statistical model with $R_{\text {adj }}^{2}=0.548$.

Conclusion: Chemical shift encoding-based water-fat MRI could detect changes in muscle composition by quantifying muscular fat that correlates well with both extensor and flexor relMVIC of the thigh. Our results help to initiate early, individualised treatments to maintain or improve muscle function in subjects who do not or not yet show pathological fatty muscle infiltration.

Keywords: Healthy volunteers, Magnetic resonance imaging, Muscle contraction (isometric), Muscle strength, Thigh

\footnotetext{
* Correspondence: stephanie.inhuber@tum.de

'Department of Sport and Health Sciences, Technische Universität München,

Georg-Brauchle-Ring 60/62, 80992 Munich, Germany

Full list of author information is available at the end of the article
} 


\section{Key points}

- Magnetic resonance imaging detects changes in muscle composition by quantifying muscular fat.

- Muscular fat correlates well with extensor and flexor strength at the thigh.

- Muscular fat, not cross-sectional area, can predict muscle strength in thigh muscles.

- The interaction between muscular fat and strength could become the basis for a biomarker for muscle quality and function.

\section{Background}

Previous research has demonstrated alterations in fat composition of thigh muscles due to various pathological conditions primarily when they are already in a chronic stage, including musculoskeletal disorders, metabolic diseases, and neuromuscular diseases (NMD) [1-9]. Furthermore, physical exercise and training have shown to entail measurable changes in the thigh musculature [10-12]. Such alterations can nowadays be captured non-invasively by means of imaging, with magnetic resonance imaging (MRI) being at the forefront particularly thanks to the ability to perform qualitative and quantitative assessments of the human body fat composition in vivo [13].

For the purpose of assessing muscle fat composition, anatomical T1- and T2-weighted MRI is conventionally applied [13]. Based on anatomical imaging, the crosssectional area (CSA) of muscles can be calculated, which can be used as a structural measure of muscle hypertrophy or atrophy [14-16]. More advanced MRI-based methods including magnetic resonance spectroscopy and chemical shift encoding-based water-fat imaging enable the extraction of parameters like the proton density fat fraction (PDFF) [13, 17]. In contrast to conventional T1- and T2-weighted MRI, chemical shift encodingbased water-fat MRI allows for robust quantitative and, thus, more objective evaluation of muscle composition whilst simultaneously enabling anatomical assessment [18-20]. In this context, thigh muscles represent a region of high interest for MRI investigation thanks to good magnetic field homogeneity, minimum motion artefacts, and the knowledge about disease-characteristic features of some pathologies in these muscles. Additionally, it is possible to perform precise strength measurements at thigh muscles, thus allowing for direct links to changes in quantitative MRI to provide insights into muscle quality and (dys-)function [21].

However, evidence of association between thigh muscle fat composition and muscle strength is scarce [22-25]. Inverse relationships were reported for the PDFF and strength at the thigh in patients with NMD $[1,8]$. However, whether the PDFF of extensor and flexor thigh muscles improves the prediction of thigh isometric strength in extension and flexion beyond the
CSA is not clear. To confirm a close relationship between thigh muscle PDFF and strength is clinically important. The thigh muscles are one of the largest muscle groups of the human body and thus important target muscles to diagnose and monitor different diseases affecting local and whole-body muscle pathologies such as knee osteoarthritis, NMD, sarcopenia, and tumour cachexia. Detecting and understanding changes in thigh muscle quality, which are based on muscle fat compositions that correlate with muscle strength, could support initiating early and individualised treatment protocols to maintain or improve muscle function prior to clinical diagnosis. Therefore, we investigated the association of thigh muscle fat with isometric strength measurement using chemical shift encodingbased water-fat MRI and a rotational dynamometer in healthy volunteers. The hypothesis is that thigh muscle PDFF improves the prediction of muscle strength measurements beyond muscle CSA.

\section{Methods \\ Subjects}

Twenty healthy volunteers (10 females; median age 27 years, range 22-41 years) were recruited. Age between 20 and 45 years and body mass index (BMI) between 23 and $33 \mathrm{~kg} / \mathrm{m}^{2}$ were defined as inclusion criteria to reflect the average population with a rather broad BMI range (median BMI of the study population $26.7 \mathrm{~kg} / \mathrm{m}^{2}$, range $22.2-31.8$ $\mathrm{kg} / \mathrm{m}^{2}$ ). Completing the international physical activity questionnaire in short-form ensured that all subjects had a moderate level of physical activity (referring to the scoring protocol: 600-1500 metabolic equivalent of task-min/week) $[26,27]$. Exclusion criteria were (1) any history of highperformance sports, (2) any history of metabolic diseases, NMD, previous knee or thigh muscle injuries, (3) general contraindications for MRI (e.g., cochlear implants), and (4) implanted foreign bodies at the level of the upper leg.

MRI and strength measurements were scheduled within 5 days to avoid any mismatch between the measured strength and fat parameters as obtained by MRIrelated analyses. In case that both parts of the measurement took place on the same day, MRI was carried out before strength measurements with the intent to not capture potential modifications in muscular structures due to strength measurement.

The study was approved by the local institutional review board and conducted in accordance with the Declaration of Helsinki. All subjects gave written informed consent on MRI examinations, isometric strength measurements, and publication of identifying images prior to participation in the study. Data are accessible upon request.

\section{MRI}

Subjects underwent 3-T MRI of the bilateral thigh muscles (Ingenia, Philips Healthcare, Best, The Netherlands). 
Scanning was performed in supine position using a built-in-the-table 12-channel posterior coil and a 16channel anterior coil.

An axially-prescribed, six-echo three-dimensional spoiled gradient-echo sequence was acquired for chemical shift encoding-based water-fat separation with the following parameters: repetition time (TR)/echo time $(\mathrm{TE}) \mathrm{min} / \Delta \mathrm{TE}=$ $6.4 / 1.1 / 0.8 \mathrm{~ms}$; field of view $220 \times 401 \times 252 \mathrm{~mm}^{3}$; acquisition matrix $68 \times 150$, voxel size $3.2 \times 2.0 \times 4.0 \mathrm{~mm}^{3}$; frequency encoding direction left-to-right $\mathrm{L} / \mathrm{R}$, no sensitivity encoding. Scan time was 1 min and $25 \mathrm{~s}$ per stack. The six echoes were acquired in a single TR using non-flyback (bipolar) read-out gradients with two axial stacks being obtained consecutively to cover the entire thigh region from the hip down to the superior edge of the patella. A flip angle of $3^{\circ}$ was used to minimise $\mathrm{T} 1$ bias effects $[18,28]$.

\section{Imaging-based fat quantification}

The imaging data were first processed by applying a phase error correction and a complex-based water-fat decomposition considering a pre-calibrated seven-peak fat spectrum and a single $\mathrm{T} 2 *[29,30]$. We used the multi-echo mDIXON fat quantification routine of the vendor. The PDFF maps were computed as the ratio of the fat signal over the sum of fat and water signals. The axial PDFF maps of both stacks of each subject were stored for segmentation.

\section{Segmentation}

Segmentations of the quadriceps femoris muscles and ischiocrural muscles of both sides were performed within the ten most central slices depicting the thigh muscles (Fig. 1). Segmentations were done on the PDFF maps using MITK (http://mitk.org/wiki/The_Medical_ Imaging_Interaction_Toolkit_(MITK); German Cancer Research Center, Division of Medical and Biological Informatics, Medical Imaging Interaction Toolkit, Heidelberg, Germany). The thigh muscles were manually segmented on the first, fifth, and tenth slice. Polygonal regions of interest (ROIs) were carefully placed in these axial slices. We then used the two-dimensional interpolation tool of MITK to obtain segmentations of the remaining axial slices. These segmentations were manually corrected. The ROIs were placed at the outer

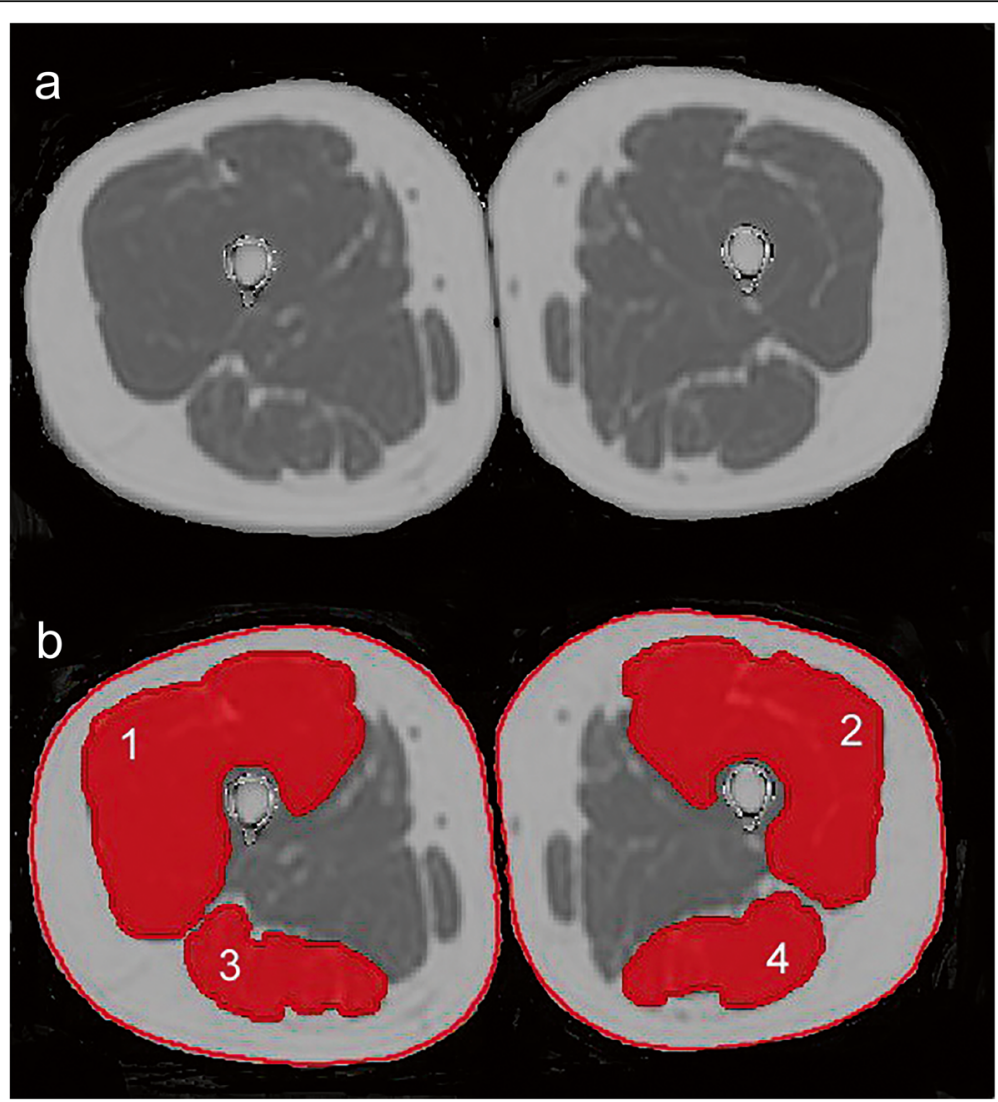

Fig. 1 Chemical shift encoding-based water-fat magnetic resonance imaging (MRI) and placement of regions of interest (ROIs). a Representative proton density fat fraction (PDFF) map. b PDFF map with superimposition of manually segmented muscle compartments defined as ROls: (1) right quadriceps muscle, (2) left quadriceps muscle, (3) right ischiocrural muscles, and (4) left ischiocrural muscles. The red lines around the thigh represent the segmentation of the entire thigh contour 
muscle contour whilst carefully avoiding the inclusion of subcutaneous fat or muscle fat interfaces [2, 22]. Subsequent to ROI placements, the CSA (in $\mathrm{mm}^{2}$ ) and PDFF (in \%) of the quadriceps and ischiocrural muscles were extracted separately for both sides by averaging the respective values obtained from the ten consecutive slices per side. Additionally, the entire thigh contour was segmented in the same ten slices (Fig. 1), followed by calculation of the mean thigh CSA per subject. A relative CSA (relCSA, in \%) of quadriceps and ischiocrural muscles was determined by dividing the respective muscle CSA by the entire thigh CSA. All segmentations were performed by a radiologist with 8 years of experience in musculoskeletal imaging. Good reproducibility of measurements following this approach was previously reported [22].

\section{Isometric muscle strength measurements}

The maximum voluntary isometric contraction (MVIC) in single-joint knee extension and flexion was measured at the thigh bilaterally with a rotational dynamometer (IsoMed 2000, D\&R Ferstl GmbH, Hemau, Germany). Substantiated by measuring the isometric peak torque in Newton per metre (in $\mathrm{N}^{*} \mathrm{~m}$ ), the MVIC was produced in knee extension at $60^{\circ}$ and knee flexion at $35^{\circ}$, which are the joint angles with the ideal muscular strength-length relationship to anticipate the real MVIC [31-34]. At the beginning of the measuring visit, the isokinetic rotational dynamometer was calibrated exactly on subjects' individual body dimension. Subjects were seated in an upright sitting position and were fixed by hip and shoulder belts as well as shoulder pads, and two adjustable straps were used to fix the leg at the pad of the lever arm in the correct measuring position (Fig. 2).

Following the individual calibrating, the subjects had to perform a standardised warm-up exercise. After 10 min on a cycling ergometer at $70-80 \mathrm{rpm}$ (revolutions per minute) to activate the cardiovascular system, the volunteers were seated in the rotational dynamometer where ten dynamic repetitions with moderate intensity were performed. In the fixed measuring position, the subjects' task was to extend or to flex the knee against the measuring pad on the back or front side of the lower leg with the individual maximum contraction of quadriceps or ischiocrural muscles. MVIC of each direction of movement (extension/flexion) was collected three times with $3 \mathrm{~min}$ of recovery in between, and the best value of muscle flexion and extension maximum isometric torque (in $\mathrm{N}^{*} \mathrm{~m}$ ) was respectively taken for data analysis $[2,22]$. The choice of the starting leg and the direction of movement were randomised.

\section{Strength data collection method}

The force transducer is located in the inside of the rotational lever arm of the used rotational dynamometer to

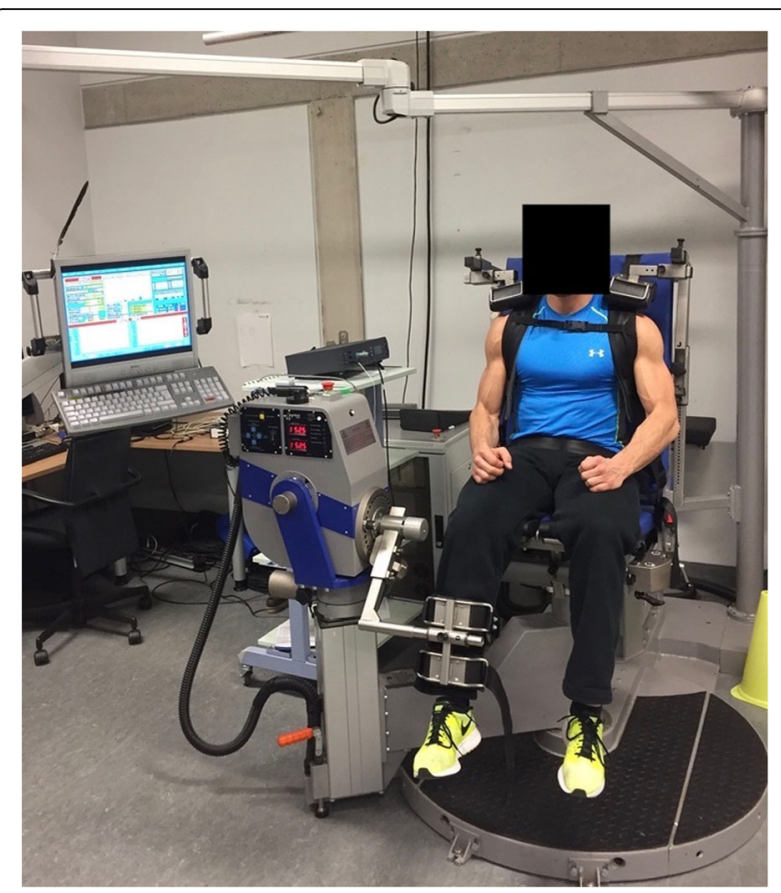

Fig. 2 Setup for measurements of the maximum voluntary isometric contraction (MVIC) with a rotational dynamometer

transfer the measured values of maximum isometric torque to the software (proEMG, Prophysics AG, Switzerland). The value the tested leg produces whilst sitting in a resting position caused by the gravity was measured in each testing step. The measured absolute extension and flexion MVICs (in $\mathrm{N}^{*} \mathrm{~m}$ ) were adjusted for the individual BMI to obtain a relative MVIC (relMVIC, in $\mathrm{N}^{*} \mathrm{~m}^{3} / \mathrm{kg}$ ) for the left and right thigh muscles.

\section{Reproducibility of muscle strength measurements}

All participants performed at least one initial training session. The visit's aim was to become familiar with the measuring procedure and to train to afford and activate the maximum isometric strength. To ensure the reproducibility of strength measurements, each subject performed at least five to eight repetitions with a MVIC and $3 \mathrm{~min}$ of rest in between. A verifiable increase and decrease of the measured values was assured as the decisive factor for a potentially needed second training visit or the testing appointment. There were at least 3 days of rest between the visits and subjects were instructed to come to the measurements totally recovered (no physical activity the 2 days before visits).

\section{Statistical analyses}

SPSS (version 20.0; IBM SPSS Statistics for Windows, Armonk, NY, USA) was used for all statistical analyses and generation of graphs. The level of statistical significance was set at $p<0.05$ (two-sided). The Kolmogorov- 
Smirnov test indicated normal distribution of BMI, PDFF, relCSA, and relMVIC values, but not of age.

First, mean \pm standard deviation was calculated for PDFF, relCSA, and relMVIC, separately for male and female subjects as well as for the right and left thigh muscles. Comparisons of these measures between genders were performed by using unpaired $t$-tests. Furthermore, Pearson correlation coefficients $(r)$ were calculated between PDFF, relCSA, and relMVIC. Using these parameters, multivariate regression models were calculated to identify significant predictors of thigh muscle strength. Parameters were included in the regression models for $p<0.05$.

\section{Results}

\section{Proton density fat fraction and cross-sectional area measurements}

Mean muscle PDFF was lower in males than females in the left and right quadriceps muscles $(2.7 \pm 1.3 \%$ versus $3.6 \pm 1.3 \%, p=0.162 ; 1.7 \pm 1.3 \%$ versus $2.7 \pm 1.3 \%, p=0.105$ ) and ischiocrural muscles $(3.2 \pm 1.6 \%$ versus $4.6 \pm 2.0 \%$, $p=0.091 ; 2.6 \pm 1.9 \%$ versus $4.9 \pm 2.4 \%, p=0.025$; Table 1 ). There were no significant differences in age or BMI between males and females ( $p=0.280$ and 0.684 , respectively).

Males showed greater relCSA than females in the quadriceps muscles $(26.5 \pm 4.0 \%$ versus $21.3 \pm 4.1 \%, p=0.010$; $25.8 \pm 4.2 \%$ versus $21.8 \pm 3.7 \%, p=0.035)$ and ischiocrural muscles at both sides $(8.3 \pm 2.4 \%$ versus $6.0 \pm 2.4 \%$, $p=0.045 ; 7.5 \pm 3.0 \%$ versus $6.6 \pm 1.6 \%, p=0.418$; Table 1 ). Quadriceps muscles had a bigger relCSA than ischiocrural muscles for males and females (Table 1).

Table 1 Proton density fat fraction (PDFF), relative crosssectional area (relCSA), and relative maximum voluntary isometric contraction (relMVIC) in extension and flexion

\begin{tabular}{lllll}
\hline & & Males & Females & $p$ value \\
\hline PDFF and relCSA & & & & \\
PDFF quadriceps (\%) & Left & $2.7 \pm 1.3$ & $3.6 \pm 1.3$ & 0.162 \\
& Right & $1.7 \pm 1.3$ & $2.7 \pm 1.3$ & 0.105 \\
PDFF ischiocrural (\%) & Left & $3.2 \pm 1.6$ & $4.6 \pm 2.0$ & 0.091 \\
& Right & $2.6 \pm 1.9$ & $4.9 \pm 2.4$ & 0.025 \\
relCSA quadriceps (\%) & Left & $26.5 \pm 4.0$ & $21.3 \pm 4.1$ & 0.010 \\
relCSA ischiocrural (\%) & Right & $25.8 \pm 4.2$ & $21.8 \pm 3.7$ & 0.035 \\
& Left & $8.3 \pm 2.4$ & $6.0 \pm 2.4$ & 0.045 \\
ReIMVIC in extension and flexion & Right & $7.5 \pm 3.0$ & $6.6 \pm 1.6$ & 0.418 \\
relMVIC in extension $\left(\mathrm{N}^{*} \mathrm{~m}^{3} / \mathrm{kg}\right)$ & Left & $8.0 \pm 1.2$ & $5.9 \pm 1.0$ & $<0.001$ \\
& Right & $8.8 \pm 1.3$ & $6.4 \pm 0.9$ & $<0.001$ \\
relMVIC in flexion $\left(\mathrm{N}^{*} \mathrm{~m}^{3} / \mathrm{kg}\right)$ & Left & $4.0 \pm 0.6$ & $3.0 \pm 0.5$ & $<0.001$ \\
& Right & $4.3 \pm 0.6$ & $2.7 \pm 0.7$ & $<0.001$ \\
\hline
\end{tabular}

Data are expressed as mean \pm standard deviation

\section{Muscle strength measurements}

RelMVIC in extension was higher in males compared to females in the left $\left(8.0 \pm 1.2 \mathrm{~N}^{*} \mathrm{~m}^{3} / \mathrm{kg}\right.$ versus $5.9 \pm 1.0$ $\left.\mathrm{N}^{*} \mathrm{~m}^{3} / \mathrm{kg}, \quad p<0.001\right)$ and right quadriceps muscles $\left(8.8 \pm 1.3 \mathrm{~N}^{* \prime} \mathrm{m}^{3} / \mathrm{kg}\right.$ versus $6.4 \pm 0.9 \mathrm{~N}^{* \prime} \mathrm{m}^{3} / \mathrm{kg}, p<0.001 ;$ Table 1). Similarly, males had greater relMVIC in flexion than females in the left $\left(4.0 \pm 0.6 \mathrm{~N}^{*} \mathrm{~m}^{3} / \mathrm{kg}\right.$ versus $3.0 \pm$ $\left.0.5 \mathrm{~N}^{*} \mathrm{~m}^{3} / \mathrm{kg}, p<0.001\right)$ and right ischiocrural muscles $\left(4.3 \pm 0.6 \mathrm{~N}^{*} \mathrm{~m}^{3} / \mathrm{kg}\right.$ versus $2.7 \pm 0.7 \mathrm{~N}^{*} \mathrm{~m}^{3} / \mathrm{kg}, p<0.001$; Table 1). Quadriceps muscles showed approximately twice as much relMVIC than ischiocrural muscles at both thighs in males (mean quadriceps $8.4 \pm 1.2 \mathrm{~N}^{*} \mathrm{~m}^{3} / \mathrm{kg}$ versus mean ischiocrural $4.1 \pm 0.6 \mathrm{~N}^{*} \mathrm{~m}^{3} / \mathrm{kg}$ ) and females (mean quadriceps $6.2 \pm 1.0 \mathrm{~N}^{*} \mathrm{~m}^{3} / \mathrm{kg}$ versus mean ischiocrural $2.9 \pm 0.6 \mathrm{~N}^{*} \mathrm{~m}^{3} / \mathrm{kg}$; Table 1$)$.

\section{Correlations and multivariate regression models}

Significant correlations were revealed between the relMVIC in extension and the PDFF of the left $(r=-0.649, p=0.002)$ and right quadriceps muscle $(r=-0.612, p=0.004$; Table 2 , Fig. 3). RelMVIC in flexion was correlated significantly with the PDFF of the left $(r=-0.446, p=0.049)$ and right ischiocrural muscles $(r=-0.676, p=0.001$; Table 2, Fig. 3).

Furthermore, the relMVIC in extension was significantly associated with the relCSA of the left $(r=0.585$, $p=0.007)$ and right quadriceps muscle $(r=0.448$, $p=0.048$; Table 2, Fig. 4). There was no significant correlation found between the relMVIC in flexion and relCSA of the ischiocrural muscles for the left and right sides (Table 2, Fig. 4).

PDFF, but not relCSA, was a statistically significant ( $p=0.001$ to 0.049$)$ predictor of relMVIC in multivariate regression models, except for left-sided relMVIC in extension. In this case, PDFF $(p=0.005)$ and relCSA $(p=0.015)$ of the quadriceps muscles contributed significantly to the statistical model with $R_{\text {adj }}^{2}=0.548$.

Table 2 Correlation between the proton density fat fraction (PDFF) or relative cross-sectional area (relCSA) and relative maximum voluntary isometric contraction (relMVIC) in extension and flexion (males and females together)

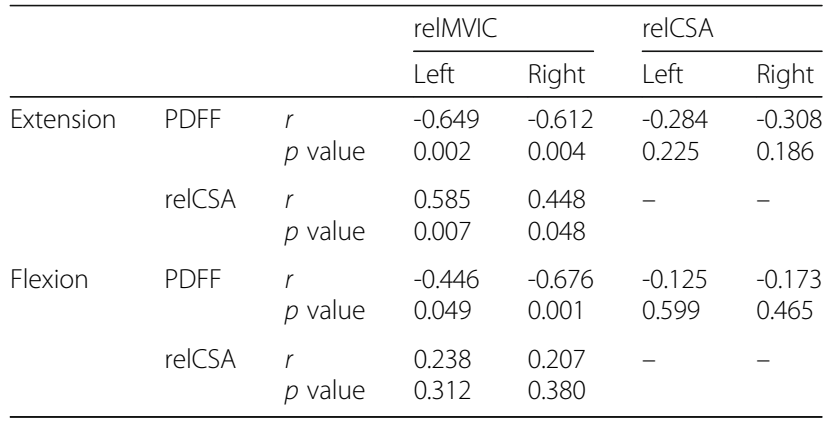



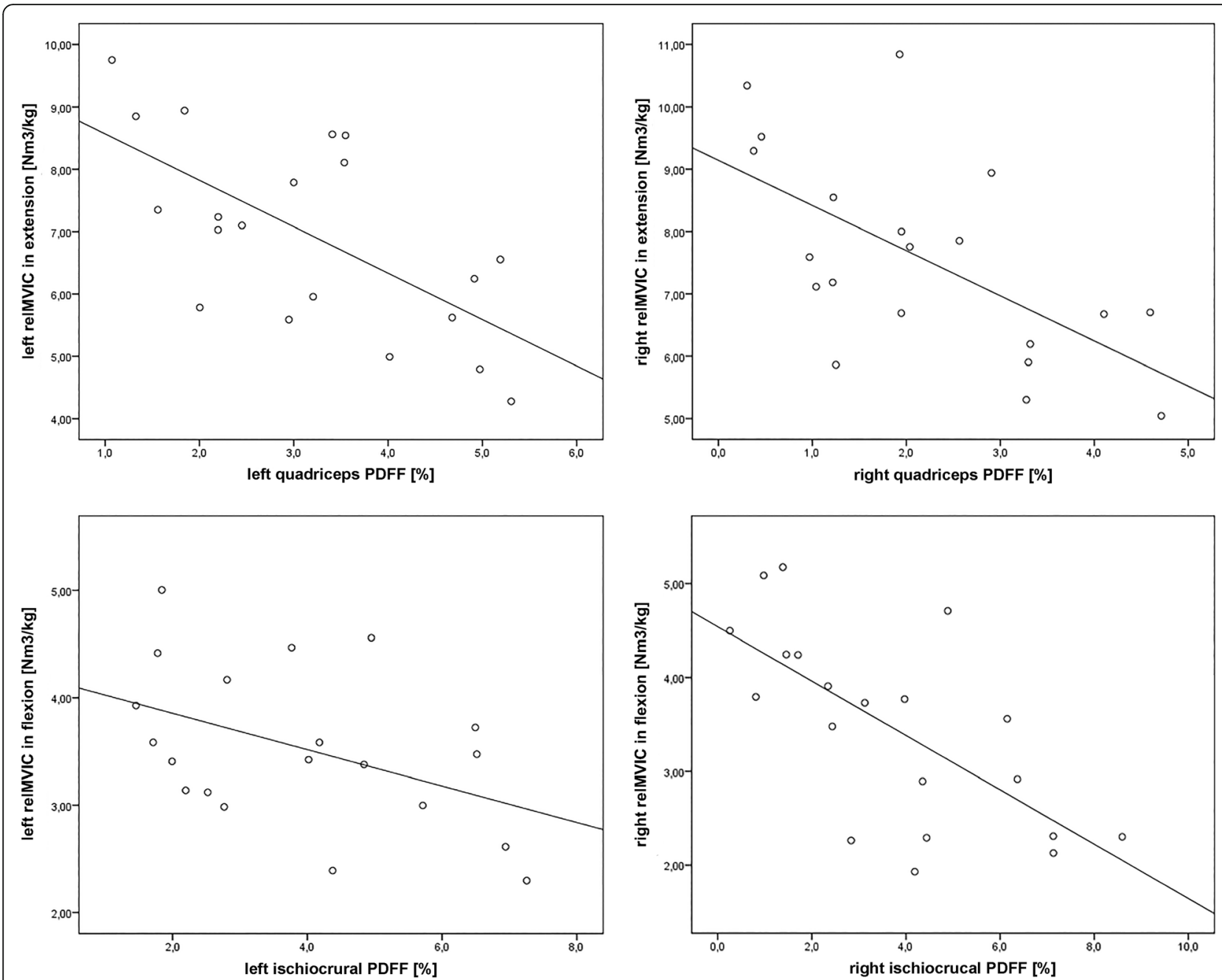

Fig. 3 Correlation between the proton density fat fraction (PDFF) and relative maximum voluntary isometric contraction (relMVIC). Plots showing the association between the left or right relMVIC in extension or flexion (in $\mathrm{N}^{*} \mathrm{~m}^{3} / \mathrm{kg}$ ) and the PDFF (in \%) of the left or right quadriceps or ischiocrural muscles

\section{Discussion}

This study used chemical shift encoding-based water-fat MRI at the thigh in healthy volunteers to extract the PDFF and relCSA of quadriceps and ischiocrural muscles bilaterally and a rotational dynamometer to assess relMVIC in extension and flexion. Muscle PDFF was a better predictor of the relMVIC than the relCSA. We observed significant differences in the relCSA of ischiocrural and quadriceps muscles, but also in the PDFF of ischiocrural muscles between males and females.

Grimm et al. [3] recently reported averaged PDFF values between 5.6 and $6.9 \%$ in healthy young males; however, in their investigation, PDFF measurements were derived from all thigh muscles and without distinct exclusion of intermuscular tissue. Specifically for the quadriceps muscles, a previous study [22] reported a mean intramuscular PDFF of $4.02 \%$ in a cohort of healthy males. Schlaeger et al. [2] performed the segmentation of individual thigh muscles as part of the MyoSegmenTUM_thigh database, with average PDFF values of $3.71 \%$ and $3.93 \%$ for the right and left quadriceps muscle as well as $4.38 \%$ and $4.44 \%$ for the right and left ischiocrural muscles by also focusing on healthy males and females. However, a distinction between genders has not been achieved in their study because only three healthy females were included in total [2]. Thus, the PDFF values obtained in the present study seem to be principally in the range of previously reported values.

Alterations in fat composition of thigh muscles have been shown in the context of diseases such as musculoskeletal disorders, metabolic diseases, and NMD [1-9]. Specifically, patients suffering from different types of muscular dystrophy, Pompe disease, sarcopenia, osteoarthritis, or type 2 diabetes mellitus showed increased PDFF or intramuscular fat when compared to healthy controls [1-9]. Although it has been hypothesised that 

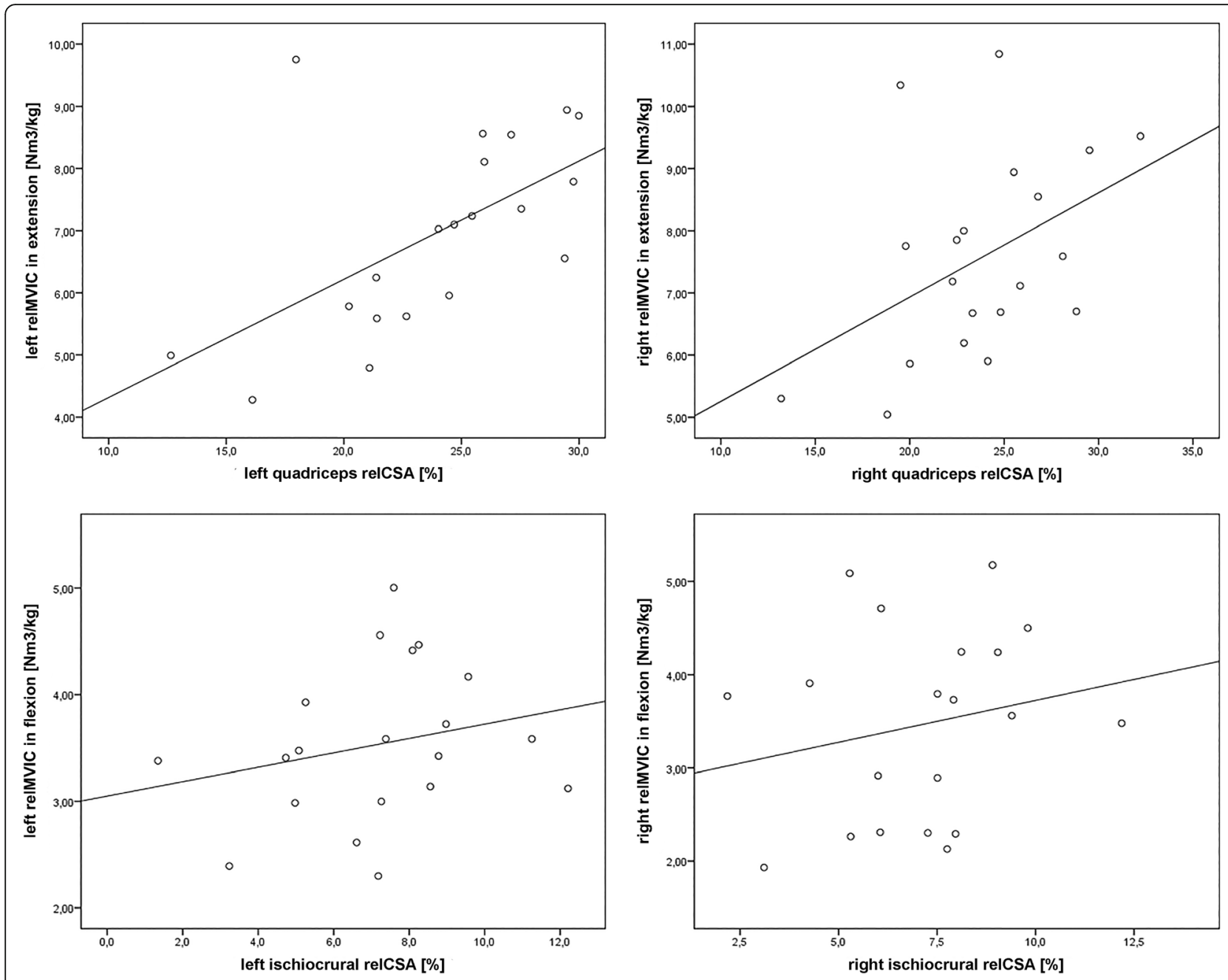

Fig. 4 Correlation between the relative cross-sectional area (relCSA) and relative maximum voluntary isometric contraction (relMVIC). Plots showing the association between the left or right relMVIC in extension or flexion (in $\mathrm{N}^{*} \mathrm{~m}^{3} / \mathrm{kg}$ ) and the relative CSA (in \%) of the left or right quadriceps or ischiocrural muscles

increased fatty infiltration or transformation of thigh muscles is related to decrease in muscle strength [21, 23], studies correlating parameters such as the PDFF or absolute or relative CSA of thigh muscles with objective measurements of muscle strength are rare. An inverse relationship was revealed between the PDFF and strength at the thigh in patients with NMD [1, 8]. In these studies, the obtained strength measurements were acquired with a handheld myometer $[1,8]$; however, the usage of more objective devices to test muscle strength, such as isokinetic dynamometers that enable robust assessments of the muscle strength of specific functional muscle groups, is still largely missing to evaluate the association between fat components and thigh muscle function. One previous study [22] focusing on the fatstrength interaction of quadriceps muscles by only testing a small cohort of healthy subjects used an isokinetic dynamometer for isometric strength measurement in knee extension and indicated that the quadriceps intermuscular adipose tissue fraction and intramuscular PDFF correlate significantly with physical strength, represented by the MVIC.

The present study confirms this first evidence of an interaction between muscular fat and strength by examining the associations between the PDFF and relMVIC at the thigh in healthy subjects and by showing not only significant correlations for the PDFF of the quadriceps muscles but also for ischiocrural muscles. Moreover, we were able to show that, in contrast to the relCSA, the PDFF of the quadriceps and ischiocrural muscles were significantly associated bilaterally with relMVIC in knee extension and flexion.

These new insights in the interactions of fat and strength parameters in human muscles demonstrate the importance of muscle quality, consisting of the individual number of muscular contractile elements, the specific fatty 
infiltration as well as the strength capacity [21]. The muscle quality might be able to predict and flesh out muscle (dys-)function a lot better than the relCSA does. Of note, there is no significant correlation between the relMVIC in flexion and the relCSA of the ischiocrural muscles for the left and right side. The anatomical difference (quadriceps muscles: one-joint muscles; ischiocrural muscles: multi-joint muscles) as well as the variations concerning the muscular structure and activation should be considered when interpreting this finding [35]. To specify the knowledge of muscle quality and (dys-)function in the future, further studies are needed to include and prove the meaning of the muscle volume and in particular the physiological CSA, the probably most powerful predictors concerning muscular strength [36-38].

Our observations seem to be in accordance with the finding of paraspinal PDFF being significantly correlated with the relMVIC in extension and flexion of the trunk, whereas paraspinal mean CSA only showed significant correlations with relMVIC in flexion in a previous study [39]. Furthermore, the suggested superiority of PDFF over relCSA for the prediction of muscle strength seems to complement previous work at the thigh region derived from patients with pathology, demonstrating negative correlations between the PDFF and muscle strength and indicating that muscle fat composition rather than muscle size correlates with knee extensor strength $[5,7-$ $9,40,41]$.

It is important to now have evidence of correlations and predictions of PDFF and muscle strength also in healthy subjects as they clearly differ from patients with musculoskeletal disorders, metabolic diseases, or NMD regarding the ranges of PDFF measurements. PDFF values are generally much lower, and the produced strength values are higher in healthy subjects [1-9]. Knowledge about such correlations and our prediction model in muscles that are not or not yet pathologically fatty infiltrated allows the PDFF to become a biomarker and to potentially facilitate early treatment protocols or to arrange counteracting individual interventions such as changes in lifestyle or specific training programmes with individual adapted physical activities in order to maintain or improve muscle function. Former studies dealt with the clinical evaluation of the knee joint agonistantagonist relationship [35, 37, 42-44]. Concerning knee dysfunctions and knee joint injuries, like ruptures of the anterior cruciate ligament, there is a high importance of the quadriceps-ischiocrural ratio. In our study, the connection of PDFF, relCSA, and relMVIC was as follows: in both genders, ischiocrural muscles were more infiltrated by fat than quadriceps muscles, had only about one third of the quadriceps relCSA, and produced about half of the quadriceps relMVIC. These insights pave the way for further research to prove the role of PDFF concerning muscle function and its role as an indicator to analyse and define muscle quality and fat-induced loss of muscle function as well as to develop corresponding training programmes dealing with these ratios. As a perspective, the role of physiological CSA, muscular pennation angle, and muscle volume could complement and specify muscle quality $[32,35]$.

Concerning methodology, chemical shift encodingbased water-fat MRI is confirmed as a fast method which can be added to routine MRI protocols of the thigh region to visualise and quantify muscle quality and possibly forecast deficits in muscle strength and function, leading over to subsequent specific training programmes. In this context, PDFF derived from chemical shift encoding-based water-fat MRI is a more robust and objective approach compared to the semi-quantitative, post-acquisitional analysis of conventional images [13]. However, the method requires compensation for confounding factors, reflected by $\mathrm{T}^{*}$ decay, a potential quantification bias due to multiple spectrum peaks, influence of eddy currents, and the T1 difference between fat and water compartments [28-30, 45]. Current sequences, such as the sequence used in this study, take these influencing factors into account. Regarding the muscle strength measurements, there is a need of familiarisation sessions prior to the testing visit to ensure to collect valid data. The rotational dynamometer testing procedure as well as the human ability to develop the real maximum of isometric strength are complex so that more visits are necessary to gain stable values of MVIC. Therefore, all participants performed at least one initial training session.

There are limitations to this study that we acknowledge. First, the comparatively small sample size. Future studies may enrol a larger sample size, which can be particularly realised by multicentre approaches using pre-existing and state-of-the-art imaging collected in joint databases [2]. Second, future studies may add magnetic resonance spectroscopy to explore the distribution of lipids within thigh muscles and distinctly quantify the intra- and extramyocellular lipid levels [13]. Third, our approach of ROI placement was restricted to segmenting the ten most central slices whilst previous investigations used larger extension or semi-automated algorithms $[2,22,46-48]$. However, since we only included healthy subjects who were characterised by rather homogeneous fat distributions, a manual segmentation approach of only representative slices seems to be justified $[22,46]$. Fourth, concerning the methodical way of strength measurement, there is a need of including physiological CSA, angle of pennation, and muscle volume in future studies as conclusive, modifying strength predictors and to integrate these values in prediction models $[36,49]$. Fifth, the present study did not enrol patients suffering from musculoskeletal disorders, metabolic 
diseases, or NMD whilst insights in varying muscle quality of different top-performing athletes are still missing.

In conclusion, we observed correlations between the PDFF and relCSA and relMVIC at the thigh by quantitative MRI and precise measurements by a rotational dynamometer. In contrast to relCSA, the PDFF of the quadriceps and ischiocrural muscles was significantly associated bilaterally with the relMVIC in extension and flexion. Thus, chemical shift encoding-based water-fat MRI can provide important information and may potentially track early changes in muscles not pathologically fatty infiltrated. This might help to initiate early, individualised training protocols with specific physical activities in order to maintain or improve muscle function.

\section{Abbreviations}

BMI: Body mass index; CSA: Cross-sectional area; MRI: Magnetic resonance imaging; MVIC: Maximum voluntary isometric contraction; NMD: Neuromuscular diseases; PDFF: Proton density fat fraction: relCSA: Relative CSA; RelMVIC: Relative MVIC; ROI: Region of interest; TE: Echo time; TR: Repetition time

\section{Authors' contributions}

$\mathrm{SI}, \mathrm{TB}$, and FK conceived the experiment. SI, SS, MD, and CK conducted the experiment. SI, NS, EB, DCK, JSK, TB, and AS analysed the acquired data, performed the statistical analysis, and interpreted the results. All authors read and approved the final manuscript.

\section{Funding}

The project was funded by the European Research Council (grant agreement No 677661_ProFatMRI and grant agreement No 637164_iBack), TUM Faculty of Medicine KKF grant H01, and Philips Healthcare.

\section{Availability of data and materials}

The datasets used and analysed during the current study are available from the corresponding author on request.

\section{Ethics approval and consent to participate}

The study was approved by the institutional review board and conducted in accordance with the Declaration of Helsinki.

\section{Consent for publication}

All subjects gave written informed consent on MRI examinations, isometric strength measurements, and publication of identifying images prior to participation in the study.

\section{Competing interests}

The authors declare that they have no competing interests.

\section{Author details}

'Department of Sport and Health Sciences, Technische Universität München, Georg-Brauchle-Ring 60/62, 80992 Munich, Germany. ${ }^{2}$ Department of Diagnostic and Interventional Neuroradiology, Klinikum rechts der Isar, Technische Universität München, Ismaninger Str. 22, 81675 Munich, Germany. ${ }^{3}$ Department of Diagnostic and Interventional Radiology, Klinikum rechts der Isar, Technische Universität München, Ismaninger Str. 22, 81675 Munich, Germany.

Received: 7 May 2019 Accepted: 13 September 2019

Published online: 20 November 2019

\section{References}

1. Dahlqvist JR, Vissing CR, Thomsen C, Vissing J (2014) Severe paraspinal muscle involvement in facioscapulohumeral muscular dystrophy. Neurology 83:1178-1183. https://doi.org/10.1212/wnl.0000000000000828

2. Schlaeger S, Freitag F, Klupp E et al (2018) Thigh muscle segmentation of chemical shift encoding-based water-fat magnetic resonance images: the reference database MyoSegmenTUM. PLoS One 13:e0198200. https://doi. org/10.1371/journal.pone.0198200

3. Grimm A, Meyer H, Nickel MD et al (2018) Repeatability of Dixon magnetic resonance imaging and magnetic resonance spectroscopy for quantitative muscle fat assessments in the thigh. J Cachexia Sarcopenia Muscle 9:10931100. https://doi.org/10.1002/jcsm.12343

4. Grimm A, Meyer H, Nickel MD et al (2018) Evaluation of 2-point, 3-point, and 6-point Dixon magnetic resonance imaging with flexible echo timing for muscle fat quantification. Eur J Radiol 103:57-64. https://doi.org/10.1016/ j.ejrad.2018.04.011

5. Kumar D, Karampinos DC, MacLeod TD et al (2014) Quadriceps intramuscular fat fraction rather than muscle size is associated with knee osteoarthritis. Osteoarthr Cartil 22:226-234. https://doi.org/10.1016/j.joca.2013.12.005

6. Karampinos DC, Baum T, Nardo L et al (2012) Characterization of the regional distribution of skeletal muscle adipose tissue in type 2 diabetes using chemical shift-based water/fat separation. J Magn Reson Imaging 35: 899-907. https://doi.org/10.1002/jmri.23512

7. Horvath JJ, Austin SL, Case LE et al (2015) Correlation between quantitative whole-body muscle magnetic resonance imaging and clinical muscle weakness in Pompe disease. Muscle Nerve 51:722-730. https://doi.org/10. 1002/mus.24437

8. Willis TA, Hollingsworth KG, Coombs A et al (2013) Quantitative muscle MRI as an assessment tool for monitoring disease progression in LGMD2l: a multicentre longitudinal study. PLoS One 8:e70993. https://doi.org/10.1371/ journal.pone.0070993

9. Willis TA, Hollingsworth KG, Coombs A et al (2014) Quantitative magnetic resonance imaging in limb-girdle muscular dystrophy 21 : a multinational cross-sectional study. PLoS One 9:e90377. https://doi.org/10.1371/journal. pone.0090377

10. Kubota J, Ono T, Araki M, Torii S, Okuwaki T, Fukubayashi T (2007) Nonuniform changes in magnetic resonance measurements of the semitendinosus muscle following intensive eccentric exercise. Eur J Appl Physiol 101:713-720. https://doi.org/10.1007/s00421-007-0549-x

11. Prior BM, Jayaraman RC, Reid RW et al (2001) Biarticular and monoarticular muscle activation and injury in human quadriceps muscle. Eur J Appl Physiol 85:185-190. https://doi.org/10.1007/s004210100434

12. Larsen RG, Ringgaard S, Overgaard K (2007) Localization and quantification of muscle damage by magnetic resonance imaging following step exercise in young women. Scand J Med Sci Sports 17:76-83. https://doi.org/10.1111/ j.1600-0838.2006.00525.x

13. Baum T, Cordes C, Dieckmeyer M et al (2016) MR-based assessment of body fat distribution and characteristics. Eur J Radiol 85:1512-1518. https://doi. org/10.1016/j.ejrad.2016.02.013

14. Wernbom M, Augustsson J, Thomee R (2007) The influence of frequency, intensity, volume and mode of strength training on whole muscle crosssectional area in humans. Sports Med 37:225-264. https://doi.org/10.2165/ 00007256-200737030-00004

15. Jones EJ, Bishop PA, Woods AK, Green JM (2008) Cross-sectional area and muscular strength: a brief review. Sports Med 38:987-994. https://doi.org/10. 2165/00007256-200838120-00003

16. Yamauchi K, Yoshiko A, Suzuki S et al (2017) Muscle atrophy and recovery of individual thigh muscles as measured by magnetic resonance imaging scan during treatment with cast for ankle or foot fracture. J Orthop Surg (Hong Kong) 25:2309499017739765. https://doi.org/10.1177/2309499017739765

17. Hu HH, Kan HE (2013) Quantitative proton MR techniques for measuring fat. NMR Biomed 26:1609-1629. https://doi.org/10.1002/nbm.3025

18. Karampinos DC, Yu H, Shimakawa A, Link TM, Majumdar S (2011) T (1)corrected fat quantification using chemical shift-based water/fat separation: application to skeletal muscle. Magn Reson Med 66:1312-1326. https://doi. org/10.1002/mrm.22925

19. Reeder SB, Hu HH, Sirlin CB (2012) Proton density fat-fraction: a standardized MR-based biomarker of tissue fat concentration. J Magn Reson Imaging 36:1011-1014. https://doi.org/10.1002/jmri.23741

20. Burakiewicz J, Sinclair CDJ, Fischer D, Walter GA, Kan HE, Hollingsworth KG (2017) Quantifying fat replacement of muscle by quantitative MRI in muscular dystrophy. J Neurol 264:2053-2067. https://doi.org/10.1007/ s00415-017-8547-3

21. McGregor RA, Cameron-Smith D, Poppitt SD (2014) It is not just muscle mass: a review of muscle quality, composition and metabolism during ageing as determinants of muscle function and mobility in later life. Longev Healthspan 3:9. https://doi.org/10.1186/2046-2395-3-9 
22. Baum T, Inhuber S, Dieckmeyer M et al (2016) Association of quadriceps muscle fat with isometric strength measurements in healthy males using chemical shift encoding-based water-fat magnetic resonance imaging. J Comput Assist Tomogr 40:447-451. https://doi.org/10.1097/rct.0000000000000374

23. Edmunds K, Gíslason M, Sigurðsson S et al (2018) Advanced quantitative methods in correlating sarcopenic muscle degeneration with lower extremity function biometrics and comorbidities. PLoS One 13:e0193241. https://doi.org/10.1371/journal.pone.0193241

24. Engelke K, Museyko O, Wang L, Laredo JD (2018) Quantitative analysis of skeletal muscle by computed tomography imaging-state of the art. J Orthop Translat 15:91-103. https://doi.org/10.1371/journal.pone.0193241

25. Harris-Love MO, Avila NA, Adams B et al (2018) The comparative associations of ultrasound and computed tomography estimates of muscle quality with physical performance and metabolic parameters in older men. J Clin Med 7:340. https://doi.org/10.3390/jcm7100340

26. Guedes DP, Lopes CC, Guedes JERP (2005) Reprodutibilidade e validade do Questionário Internacional de Atividade Física em adolescentes. Rev Bras Med Esporte 11:151-158. https://doi.org/10.1590/S1517-86922005000200011

27. Kurtze N, Rangul V, Hustvedt BE (2008) Reliability and validity of the international physical activity questionnaire in the Nord-Trondelag health study (HUNT) population of men. BMC Med Res Methodol 8:63. https://doi. org/10.1186/1471-2288-8-63

28. Liu CY, McKenzie CA, Yu H, Brittain JH, Reeder SB (2007) Fat quantification with IDEAL gradient echo imaging: correction of bias from T (1) and noise. Magn Reson Med 58:354-364. https://doi.org/10.1002/mrm.21301

29. Bydder M, Yokoo T, Hamilton G et al (2008) Relaxation effects in the quantification of fat using gradient echo imaging. Magn Reson Imaging 26: 347-359. https://doi.org/10.1016/j.mri.2007.08.012

30. Yu H, Shimakawa A, McKenzie CA, Brodsky E, Brittain JH, Reeder SB (2008) Multiecho water-fat separation and simultaneous R2* estimation with multifrequency fat spectrum modeling. Magn Reson Med 60:1122-1134. https://doi.org/10.1002/mrm.21737

31. Alangari AS, Al-Hazzaa HM (2004) Normal isometric and isokinetic peak torques of hamstring and quadriceps muscles in young adult Saudi males. Neurosciences (Riyadh) 9:165-170. 23377421

32. Dirnberger J, Wiesinger H-P, Kösters A, Müller E (2012) Reproducibility for isometric and isokinetic maximum knee flexion and extension measurements using the IsoMed 2000-dynamometer. Isokinet Exerc Sci 20: 149-153. https://doi.org/10.3233/ies-2012-0451

33. Maffiuletti NA, Jubeau M, Agosti F, De Col A, Sartorio A (2008) Quadriceps muscle function characteristics in severely obese and nonobese adolescents. Eur J Appl Physiol 103:481-484. https://doi.org/10.1007/s00421-008-0737-3

34. Onishi H, Yagi R, Oyama M, Akasaka K, Ihashi K, Handa Y (2002) EMG-angle relationship of the hamstring muscles during maximum knee flexion. J Electromyogr Kinesiol 12:399-406. 12223173

35. Bollinger R, Bubeck D, Sialis I, Kramer D, Lehmann E, Alt W (2017) Relationship between muscle volume and strength asymmetry in elite athletes: investigation of the quadriceps femoris and hamstring muscles. Paper presented at the 35th conference of the International Society of Biomechanics in sports, Cologne, Germany, 14-18 June 2017

36. Blazevich AJ, Coleman DR, Horne S, Cannavan D (2009) Anatomical predictors of maximum isometric and concentric knee extensor moment. Eur J Appl Physiol 105:869-878. https://doi.org/10.1007/s00421-008-0972-7

37. Ikai M, Fukunaga T (1968) Calculation of muscle strength per unit crosssectional area of human muscle by means of ultrasonic measurement. Int Z Angew Physiol 26:26-32 https://doi.org/10.1007/bf00696087

38. Maughan RW, Watson JS, Weir J (1983) Strength and cross-sectional area of human skeletal muscle. J Physiol 338:37-49. https://doi.org/10.1113/jphysiol. 1983.sp014658

39. Schlaeger S, Inhuber S, Rohrmeier A et al (2019) Association of paraspinal muscle water-fat MRI-based measurements with isometric strength measurements. Eur Radiol 29:599-608. https://doi.org/10.1007/s00330-0185631-8

40. Heymsfield SB, Gonzalez MC, Lu J, Jia G, Zheng J (2015) Skeletal muscle mass and quality: evolution of modern measurement concepts in the context of sarcopenia. Proc Nutr Soc 74:355-366. https://doi.org/10.1017/ S0029665115000129

41. Lev MH, Farkas J, Rodriguez VR et al (2001) CT angiography in the rapid triage of patients with hyperacute stroke to intraarterial thrombolysis: accuracy in the detection of large vessel thrombus. J Comput Assist Tomogr 25:520-528. https://doi.org/10.1097/00004728-200107000-00003
42. Aagaard P, Simonsen EB, Beyer N, Larsson B, Magnusson P, Kjaer M (1997) Isokinetic muscle strength and capacity for muscular knee joint stabilization in elite sailors. Int J Sports Med 18:521-525. https://doi.org/10.1055/s-2007972675

43. Holcomb WR, Rubley MD, Lee HJ, Guadagnoli MA (2007) Effect of hamstringemphasized resistance training on hamstring: quadriceps strength ratios. J Strength Cond Res 21:41-47. https://doi.org/10.1519/R-18795.1

44. Mau-Moeller A, Gube M, Felser S et al (2019) Intrarater reliability of muscle strength and hamstring to quadriceps strength imbalance ratios during concentric, isometric, and eccentric maximal voluntary contractions using the isoforce dynamometer. Clin J Sport Med 29:69-77. https://doi.org/10. 1097/jsm.0000000000000493

45. Yu H, Shimakawa A, Hines CD et al (2011) Combination of complex-based and magnitude-based multiecho water-fat separation for accurate quantification of fat-fraction. Magn Reson Med 66:199-206. https:/doi.org/10.1002/mrm.22840

46. Barnouin Y, Butler-Browne G, Voit T et al (2014) Manual segmentation of individual muscles of the quadriceps femoris using MRI: a reappraisal. J Magn Reson Imaging 40:239-247. https://doi.org/10.1002/jmri.24370

47. Karlsson A, Rosander J, Romu T et al (2015) Automatic and quantitative assessment of regional muscle volume by multi-atlas segmentation using whole-body water-fat MRI. J Magn Reson Imaging 41:1558-1569. https://doi. org/10.1002/jmri.24726

48. Morrow JM, Sinclair CD, Fischmann A et al (2014) Reproducibility, and age, body-weight and gender dependency of candidate skeletal muscle MRI outcome measures in healthy volunteers. Eur Radiol 24:1610-1620. https:// doi.org/10.1007/s00330-014-3145-6

49. Maughan RW, Weir J (1984) Muscle strength and cross-sectional area in man: a comparison of strength-trained and untrained subjects. Br J Sports Med 18:149-157. https://doi.org/10.1136/bjsm.18.3.149

\section{Publisher's Note}

Springer Nature remains neutral with regard to jurisdictional claims in published maps and institutional affiliations.

\section{Submit your manuscript to a SpringerOpen ${ }^{\circ}$ journal and benefit from:}

- Convenient online submission

- Rigorous peer review

- Open access: articles freely available online

High visibility within the field

- Retaining the copyright to your article

Submit your next manuscript at $\boldsymbol{\nabla}$ springeropen.com 